\title{
Las redes de servicios. Contribuciones en el acceso y la cobertura de salud'
} Health networks. Contribution to health coverage and access

\section{Ana Ariovich ${ }^{\mathrm{a}}$}

(D) https://orcid.org/0000-0001-6142-4841

E-mail: anariovichळyahoo.com

María Crojethovic ${ }^{\mathrm{a}}$

(D) https://orcid.org/0000-0002-4251-8532

E-mail: mcrojeœgmail.com

a Universidad Nacional General Sarmiento. Instituto del Conurbano. Buenos Aires, Argentina.

\section{Correspondencia}

Ana Ariovich

Calle Juan Maria Gutierrez, I150. Buenos Aires, Argentina. CP B1613.

\section{Resumen}

Desde un abordaje que recupera los aportes de la Teoría de las Organizaciones y de la literatura en redes, el trabajo describe y analiza las redes de servicios que operan en el sistema de salud del Gran Buenos Aires (GBA). Pone el foco en la estructura organizacional que muestran las iniciativas, a la vez que explora los mecanismos y dispositivos que despliegan para articular recursos, prestaciones y servicios dispersos en el territorio. Para la identificación y la selección de las redes se entrevistó a los Directores Ejecutivos de las cuatro Regiones Sanitarias (RS) del conurbano bonaerense (4 entrevistas). Posteriormente, se aplicó una ficha de relevamiento en cada una de las redes seleccionadas; la indagación se focalizó en cuatro grandes dimensiones derivadas de la perspectiva teórica: dimensión estructural, amplitud de la red y dimensión organizacional. Finalmente, se realizaron 18 entrevistas en profundidad a sus coordinadores. La reconstrucción y el análisis se completaron triangulando la evidencia empírica con fuentes secundarias de organismos oficiales. Las redes estudiadas alcanzan distintas escalas territoriales y se apoyan en programas de salud nacionales y/o provinciales preexistentes. Además de establecimientos de mediana y alta complejidad del subsector público, involucran - en muchos casos prestaciones del primer nivel de atención; sin embargo, la lógica organizacional se centra en el hospital. Finalmente, se observa una carencia de instancias, dinámicas y recursos formalmente destinados al funcionamiento. Esto ha implicado un alto compromiso de los equipos involucrados para poder gestionarlas y, se ha traducido en importantes limitaciones para su desarrollo y autonomía.

Palavras-chave:Servicios de Salud; Cobertura de Servicios Públicos de Salud; Sistemas de Salud; Accesibilidad a los Servicios de Salud; Organización y Administración.

1 El trabajo fue realizado en el marco del proyecto “Cobertura universal de salud y redes de servicios: encuentros y desencuentros entre la macro y la meso gestión. Un estudio de caso en el conurbano bonaerense 2008/2015” con financiamiento del FONCyT -proyecto PICT-2014-0693- 


\section{Abstract}

From an approach that recovers the contributions of the Theory of Organizations and specialized literature in networks, the work describes and analyzes the health service networks that operate in Greater Buenos Aires (GBA). It puts the focus on the organizational structure that these initiatives show, while also exploring the mechanisms and devices they deploy to coordinate resources and services scattered throughout the territory. For the identification and survey of the health services networks in the GBA, a theoretical methodological instrument was applied. Subsequently, 22 interviews were made to the referents / coordinators. The reconstruction and analysis were completed by triangulating the empirical evidence collected with secondary sources of official health agencies. The networks studied reach different territorial scales and rely on pre-existing national or provincial health programs. In addition to medium and high complexity establishments belonging to the public subsector, they involve - in many cases - first-level care services; however, the organizational logic focuses on the hospital. Finally, there is a lack of instances, dynamics and resources formally destined to the functioning of the networks. This has implied a high commitment and a great effort on the part of the teams involved to manage them and, at the same time, it has translated into important limitations for their development and autonomy. Keywords: Health Services; State Health Care Coverage; Health Systems; Health Services Accessibility; Organization and Administration.

\section{Introducción}

Las redes de servicios de salud son promovidas como una estrategia orientada a mejorar el acceso, la cobertura y los beneficios en contextos de fuerte fragmentación (Cetrángolo, 2014; OPS, 2007). En sucesivos documentos, los organismos internacionales de salud ${ }^{2}$ las han definido como modelos organizativos para articular de manera estratégica servicios de distintas jerarquías y complejidad en territorios de escala local o regional, a partir de una acción cooperativa e interdependiente (Mendes, 2013). En ellas, la atención primaria de la salud (APS) tiene un papel distintivo como espacios de coordinación de la atención entre los diferentes niveles (Harzheim, 2011; Mendes, 2013).

Este trabajo describe y analiza las redes de servicios de salud que operan en Gran Buenos Aires, ${ }^{3}$ poniendo el foco en la estructura organizacional. Además, explora los mecanismos y dispositivos que despliegan estas iniciativas para articular y coordinar recursos, prestaciones y servicios dispersos en ese territorio.

\section{La salud en el Gran Buenos Aires: reseña sobre el sistema de salud}

Asentado sobre la provisión pública, con independencia de la posesión de algún tipo de aseguramiento social o privado, el sector de la salud en la Argentina se encuentra conformado por tres subsectores: el estatal, el de la seguridad social y el privado. Mientras que los trabajadores formales utilizan las instituciones de la seguridad social,4,5 los principales beneficiarios del sector público son

\footnotetext{
2 Estos organismos han sostenido que las Redes Integradas de Servicios (RIS) y los Sistemas Integrados de Servicios de Salud (SISS) posibilitan la gestión y entrega de servicios de forma que las personas reciban un continuo de servicios preventivos y curativos, de acuerdo a sus necesidades a lo largo del tiempo y a través de los diferentes niveles del sistema (OPS 2008).

3 En este trabajo denominamos Gran Buenos Aires solo al conjunto integrado por los 24 partidos de la Provincia de Buenos Aires, más cercanos a la Ciudad Autónoma de Buenos Aires.

4 En Argentina, la seguridad social suele definirse como el conjunto de programas y políticas que tienen como objetivo cubrir riesgos asociados al trabajo formal. Se constituyen con el fin de proveer certidumbre y asegurar a trabajadores formales frente al riesgo de pérdidas de ingresos o shocks de gastos asociados con el retiro en la vejez, invalidez, enfermedad, accidentes, fallecimiento o situación de desempleo (Cursio, 2011). En Brasil, es un concepto más amplio, que abarca no sólo la parte previsional y la salud, sino también la asistencia social. Vianna (200o, p. 58, traducción propia) destaca que "no todos los países cuya protección social es avanzada - desde el punto de vista valorativo - consideran como seguridad social idéntico conjunto de acciones”.

5 Algunas obras sociales utilizan (por convenio o por sistema de recupero) a los efectores públicos de salud como prestadores para brindar atención a sus afiliados
} 
aquellas personas que no cuentan con obras sociales ni cobertura privada. ${ }^{6}$

Diversos estudios señalan cómo esta diagramación brinda un alto nivel de cobertura a su población. A pesar de ello, también mencionan la baja articulación entre sus subsectores y las complejas interacciones que se producen entre un conjunto de actores con desiguales intereses (como prestadores de servicios asistenciales, financiadores o empresas de seguros médicos, laboratorios de especialidades médicas, etc. que conforman el subsector privado) (Cetrángolo, 2014). A su vez, el régimen de gobierno federal genera no sólo intervenciones y fragmentación entre la Nación y las provincias, sino también entre las provincias y entre estas últimas y los municipios, profundizando la fragmentación general del sector (Acuña; Chudnovsky, 2002).

En la Provincia de Buenos Aires, el subsector estatal se fue constituyendo por una progresiva transferencia de establecimientos desde la jurisdicción nacional hacia las provincias y, por el traspaso de los centros de salud provinciales a la órbita de los municipios (Acuña; Chudnosvky, 2002; Cetrángolo 2014). Hecho que no estuvo acompañado por la definición de las responsabilidades a cumplir por cada nivel gubernamental y que operó sobre municipios con capacidades institucionales y recursos heterogéneos, dando lugar a brechas de desigualdad entre los territorios locales en el acceso y en las modalidades de la atención (Chiara; Di Virgilio; Ariovich, 2010).

El subsector público bonaerense, además, fue delineando su política sanitaria con una organización territorial que contempló doce regiones sanitarias (Ley 7016 del año 1965); cada una de estas comenzó a representar un espacio desconcentrado del estado provincial para la coordinación técnicoadministrativa de la política sanitaria (Chiara, 2012).

En suma, en el territorio bonaerense la gestión y la prestación de servicios sanitarios transcurre en un escenario fuertemente fragmentado. A su vez, al interior de la esfera estatal, el diseño de las políticas y la provisión de servicios descansan en una estructura organizativa en la que se combinan desarticuladamente los niveles nacional, provincial y municipal con consecuencias en el acceso, la cobertura y la calidad de la atención.

\section{El aporte de las redes a los problemas de acceso a la atención y cobertura}

La noción de redes en salud descansa sobre una premisa general: garantizar el acceso de la población a todos los niveles de atención, en especial cuando los sistemas de salud están fuertemente fragmentados (Mendes, 2013; Shortell et al., 1993). Aglutinar al sistema y mejorar tanto el alcance como la eficiencia de las intervenciones, a partir de una configuración coordinada de servicios para una población determinada, es la mayor potencialidad que ha sido atribuida a las redes -desde la literaturaen términos de accesibilidad a la salud (Dabas; Perrone, 1999; Shortell et al., 1993).

Si bien varias propuestas circunscriben el problema del acceso a la trayectoria que va desde la búsqueda hasta el inicio de la atención (Adaszko, 2011; Donabedian, 2003; Rodriguez; Roldan, 2008), otros enfoques otorgan a la problemática un alcance mayor. En estos, el acceso además abarca factores como las necesidades de salud que dan origen a la demanda de atención, el proceso de toma de decisiones que antecede a la búsqueda de la misma, la continuidad en la utilización de las prestaciones y los resultados luego del contacto inicial con los servicios (Andersen, 1995; Levesque; Harris; Russell, 2013). Desde esta perspectiva la accesibilidad no puede ser entendida sólo como la obtención de atención. El acceso también contempla la utilización oportuna y continua de servicios de tratamiento, de prevención y de promoción, de manera coordinada entre los distintos niveles de complejidad del sistema, acorde a las necesidades de la comunidad (OPS, 2008).

En términos ideales, la cobertura refiere a que las comunidades tengan acceso de acuerdo a sus necesidades a servicios integrales, adecuados, oportunos y de calidad, y a medicamentos; esta puede

6 Algunos beneficiarios de obras sociales o sistemas prepagos que brindan pocas o malas prestaciones optan por utilizar el subsector público. 
ser pública, privada y/o de la seguridad social 7 . A la vez, debe asegurarse que el uso de esos servicios no exponga a los usuarios a dificultades financieras (OPS, 2014). En cambio, la cobertura efectiva en salud puede ir desde la existencia de un mínimo, al alcance de todos, hasta una cobertura amplia, suficiente e igualitaria (Crojethovic, 2016). A pesar de esto, muchos países de la región no llegan a dicha cobertura mínima, sino que resulta insuficiente y desigualmente distribuida (Cetrángolo, 2014).

Con el propósito de facilitar el acceso a la atención integral y un mayor alcance en la cobertura a una población definida (Mendes, 2013), las redes articulan de manera estratégica un conjunto de complejas organizaciones, con estructuras que involucran a distintos actores portadores de conocimientos específicos como profesionales, técnicos o administrativos. Esto da lugar tanto a dinámicas de poder y autoridad como a márgenes de autonomía muy diferentes a las de otros modelos organizacionales con grupos más homogéneos (Crojethovic; Ariovich, 2015). Si bien cuentan con mecanismos de normalización para coordinar las actividades de los distintos actores, no siempre poseen los dispositivos necesarios para otorgar a estos profesionales o técnicos la autonomía requerida para el desarrollo de habilidades y conocimientos expertos (Etzioni, 1975).

El modelo organizativo multi-institucional que suponen las redes de servicios se basa en mecanismos contractuales y técnicos entre las distintas organizaciones corresponsables en procesos de trabajo destinados a satisfacer necesidades de terceros. A la vez, requiere de la existencia de nodos en los que circulan decisiones, información y recursos. Las redes pueden ser especializadas o redundantes descentralizadas: en las primeras los actores acuerdan complementarse y aprovechar las facilidades de la especialización de cada parte de la red, facilitando la cooperación; en las segundas coexisten actores institucionales que producen los mismos productos o servicios de manera simultánea para reducir riesgos (Hintze, 2009).

Dicha forma de organización aporta una visión particular del territorio: más sistémica y menos fragmentada, en la que distintos flujos de interacción y complementariedad vinculan y articulan un conjunto disperso de prestaciones y efectores de diferente complejidad y/o especialización en un mismo espacio. Este puede alcanzar una escala amplia en dónde las distancias físicas y operativas se relativizan, posibilitando una continuidad adecuada a las necesidades de los procesos de atención y a la optimización de los recursos disponibles (Chiara; Ariovich, 2013).

Los desafíos de gestión que presenta la creación y el mantenimiento de las redes a lo largo del tiempo son diversos. Los más importantes están vinculados al establecimiento de reglas de actuación, a la distribución de recursos, a la definición de prioridades, a la necesidad de generar procesos de negociación o consenso y a la construcción de mecanismos colectivos de decisión (Fleury, 2002). Dicho mantenimiento debe ser pensado desde una perspectiva dinámica, lo que supone dar cuenta no sólo de su conformación y funcionamiento, sino también de sus transformaciones y, paralelamente, de considerarlas como móviles e inacabadas. Asimismo, también ha sido resaltada la importancia de los flujos constantes de información para conectar sujetos y actores al interior de la propia red, formando un conjunto articulado (Blanco, 2009). Por eso, la posibilidad de establecer redes que vinculen bien la gestión de las políticas sanitarias con el territorio y una población específica está fuertemente condicionada por el despliegue tecnológico de comunicaciones que habiliten interacciones virtuales en tiempo real (Fleury, 2002), y por el desarrollo de una red de transporte público y viario que permita sortear las barreras espaciales (Gutiérrez, 2009).

7 En el caso de la Argentina, por ejemplo, el sector de la salud comprende tres subsectores altamente desarticulados y que brindan coberturas que pueden superponerse (como vimos en el segundo apartado). Además, de un conjunto muy heterogéneo de obras sociales para los trabajadores formales o de sistemas prepagos, la población tiene acceso a las prestaciones del subsector estatal (independiente de la posesión de otro tipo de cobertura). Si bien los principales beneficiarios de los efectores estatales son los sectores de menores recursos, algunas personas optan por usar las prestaciones del subsector público debido a la poca o a la mala calidad de la oferta de salud de sus obras sociales o prepagas (Cetrángolo, 2014). 
Estos aportes nos dejan comprender que las redes de servicios de salud pueden ser entendidas como un continuo coordinado de servicios de promoción y prevención, de diagnóstico, de tratamiento, de rehabilitación y de cuidados paliativos para una población y territorios definidos. Las redes articulan estratégicamente y de manera cooperativa -a través de relaciones contractuales y técnicas y flujos de información- un conjunto de complejas organizaciones que albergan dinámicas particulares de control, poder y autonomía entre sus actores; estas pueden mostrarse móviles en el tiempo, cambiantes, inestables y/o inacabadas (Crojethovic; Ariovich, 2015).

\section{Abordaje metodológico}

Uno de los desafíos de la investigación ${ }^{8}$ que dio origen a este trabajo, fue reconocer las experiencias de redes de servicios de salud que efectivamente se encuentran en funcionamiento en el segundo nivel atención (hospitales) del Gran Buenos Aires. ${ }^{9}$ Con este propósito, se entrevistó a los Directores Ejecutivos de las cuatro Regiones Sanitarias (RS) ${ }^{10}$ que integran el territorio del conurbano bonaerense (se hicieron 4 entrevistas en profundidad). La información obtenida en esta instancia posibilitó identificar un total de 27 redes en actividad, de las cuales se seleccionaron 17 redes ( 2 nacionales, 2 provinciales y 13 regionales); los criterios para la elección de los casos a estudiar fueron construidos en base a 4 variables: tipo de cobertura (nacional, provincial, municipal), cantidad de efectores involucrados, tipo de problemática abordada (aguda o crónica) y antigüedad de la red. ${ }^{11}$

Las redes que integran el estudio pertenecen a los distintos niveles jurisdiccionales que se encuentran a cargo de la salud en Argentina (nacional, provincial o regional), ${ }^{12}$ y refieren a problemáticas tanto como agudas o crónicas.

Cuadro I - Problemáticas
\begin{tabular}{|l|l|}
\hline \multicolumn{1}{|c|}{ Agudas } & \multicolumn{1}{c|}{ Crónicas } \\
\hline Cardiopatías congénitas & $\begin{array}{l}\text { Diabetes, hipertensión arterial, } \\
\text { enfermedades cardiovasculares }\end{array}$ \\
\hline Emergencias/traslados & Nefrología \\
\hline Perinatal & Salud sexual y reproductiva \\
\hline Hemoterapia & VIH \\
\hline
\end{tabular}

El trabajo de campo se desarrolló entre julio y octubre de 2015, e implicó la aplicación de una ficha de relevamiento en cada una de las redes seleccionadas. Esta indagación focalizó en cuatro grandes dimensiones, derivadas de la perspectiva teórica abordada: dimensión estructural, amplitud de la red y dimensión organizacional. Posteriormente, se realizaron 18 entrevistas en profundidad ${ }^{13}$ a los referentes/coordinadores de las redes. Mientras que los datos del relevamiento se procesaron utilizando el programa SPSS, las entrevistas se codificaron utilizando el software AtlasTi.

La reconstrucción y análisis trianguló la información cuantitativa del relevamiento, el material de las entrevistas y fuentes secundarias de organismos oficiales (documentos e informes de avance de programas vinculados con las

\footnotetext{
8 La investigación se desarrolló en el marco del Proyecto PICT 2014 - o693: "Cobertura universal de salud y redes de servicios: encuentros y desencuentros entre la macro y la meso gestión. Un estudio de caso en el conurbano bonaerense 2008/2015”. Ambas autoras formaron parte del equipo responsable de la dirección del proyecto.

9 Cabe destacar que quedaron fuera del estudio aquellas redes que comprometían exclusivamente a efectores del primer nivel de atención. 10 Las regiones sanitarias comprenden al área geográfica en la cual se integran en red todos los organismos encargados del cumplimiento de las funciones de la salud pública en sus tres aspectos principales de protección, fomento y recuperación de la salud, y sus complementarias, que hacen al completo bienestar psíquico, físico y social de la comunidad (Ley 7016 de Regionalización Sanitaria en la provincia de Buenos Aires y decreto 3377/o6). La Provincia de Buenos Aires cuenta con 12 regiones, de las cuales hemos tomado para nuestro estudio cuatro de ellas: V, VI, VII y XII. Todas estas comprenden los 24 partidos del Gran Buenos Aires, más cercanos a la Ciudad Autónoma de Buenos Aires.

11 Las regiones sanitarias comprenden al área geográfica en la cual se integran en red todos los organismos encargados del cumplimiento de las funciones de la salud pública en sus tres aspectos principales de protección, fomento y recuperación de la salud, y sus complementarias, que hacen al completo bienestar psíquico, físico y social de la comunidad (Ley 7016 de Regionalización Sanitaria en la provincia de Buenos Aires y decreto 3377/o6).

12 Como ha sido detallado en el apartado La salud en el Gran Buenos Aires: reseña sobre el sistema de salud de este artículo.

13 Cabe señalar que para analizar la Red provincial de emergencias (Sies), se hicieron 2 entrevistas en profundidad: una primera entrevista al reciente referente de la red, y una segunda a su antecesor (debido a su trayectoria mucho más amplia en el cargo).
} 
problemáticas abarcadas por las redes, leyes nacionales y provinciales, decretos nacionales y provinciales, convenios para la implementación de las políticas/programas, y datos epidemiológicos y de desempeño de los sistemas sanitarios).

\section{Resultados}

\section{La organización de los servicios en red}

Los establecimientos que dan entidad a las redes, por lo general pertenecen al subsector público estatal. Estas nacen con el objetivo de articular recursos profesionales, insumos, información y/o tecnologías entre los hospitales (de distinto nivel jurisdiccional y/o diferente complejidad), los centros de atención primaria y/o los institutos especializados. Sin embargo, y a pesar de que no surge como una exclusión premeditada, la relación con las instituciones privadas -con y sin fines de lucro-, es casi inexistente.

En un intento por reducir la fragmentación entre la Provincia de Buenos Aires y los municipios del GBA, se incorporan hospitales públicos tanto de la provincia como municipales; aunque al quedar muy pocos bajo la órbita nacional, la relación entre los prestadores de la Nación y la Provincia de Buenos Aires es menos frecuente. En algunos casos, contemplan también centros de atención primaria, lo que les permite abordar problemáticas que demandan mayor interacción con la población local (por ejemplo, las redes de Salud Sexual y Reproductiva o las de Enfermedades Crónicas No Transmisibles).

$\mathrm{Al}$ interior de cada red, los equipos trabajan interdisciplinariamente con el propósito de complementarse y aprovechar las facilidades de la especialización de sus profesionales (Hintze, 2009), sobre todo en aquellas especialidades en las cuales hay poca disponibilidad de la oferta dentro del subsector público.

Existe cierta racionalidad por la cual los establecimientos son en su mayoría públicos. Muchas de las redes emergen de la necesidad de vincular horizontalmente a los distintos programas creados para abordar problemáticas específicas, sean nacionales o de la Provincia. Por eso, casi la totalidad de las que han sido estudiadas, organizan una especial interacción con al menos algunos de los programas previamente implementados -desde el nivel central o provincial- (ver Cuadro 1), para optimizar recursos e insumos que faciliten la atención de cada problemática y mejorar el acceso, la cobertura y los beneficios brindados. Este modo de organización que involucra a diversos programas, ha mostrado en algunas problemáticas, una contribución en la reducción de la fragmentación en los procesos de atención. Ejemplo de ello es la Red de Cardiopatías Congénitas, que logró reducir la lista de espera en las operaciones, aumentar la cantidad de intervenciones quirúrgicas, y disminuir la mortalidad por cardiopatías (Andiarena, 2018).

\section{Cuadro 2 - Programas y organismos nacionales y provinciales}

\begin{tabular}{|c|c|}
\hline Hemoterapia & $\begin{array}{l}\text { Instituto Provincial de Hemoterapia; } \\
\text { INCUCAI }\end{array}$ \\
\hline $\begin{array}{l}\text { Cardiopatías } \\
\text { Congénitas }\end{array}$ & $\begin{array}{l}\text { Programa Nacional de Cardiopatías } \\
\text { Congénitas; } \\
\text { Programa de Materno infantil de la Provincia }\end{array}$ \\
\hline ECNT & $\begin{array}{l}\text { Programa de Prevención de Epilepsia } \\
\text { (PROEPI); } \\
\text { Prevención del Asma Infantil (PROBAS), } \\
\text { Remediar }\end{array}$ \\
\hline SS y $R$ & $\begin{array}{l}\text { Programa Nacional de Salud Sexual y } \\
\text { Procreación Responsable; } \\
\text { Programa Nacional de Prevención de Cáncer } \\
\text { Cérvicouterino; } \\
\text { Programa Nacional Salud y Adolescencia; } \\
\text { Programa Nacional de Lucha contra el SIDA y } \\
\text { Enfermedades de Transmisión Sexual (ETS); } \\
\text { Programa Nacional de Asistencia para } \\
\text { medicamentos y reactivos SIDA; } \\
\text { Plan Nacional para la reducción de la } \\
\text { mortalidad materno infantil, de las mujeres y } \\
\text { las adolescentes; } \\
\text { Programa Provincial de Salud Reproductiva y } \\
\text { Procreación Responsable; } \\
\text { Dirección Provincial de Atención a las } \\
\text { Adicciones; } \\
\text { Programa Materno Infantil de la Provincia; } \\
\text { Programa de Prevención de Cáncer Genito- } \\
\text { mamario (PRoGEMA); } \\
\text { Programa Provincial de VIH-SIDA, ITS y } \\
\text { Hepatitis Virales }\end{array}$ \\
\hline
\end{tabular}




\section{Cuadro 2 - Continuación}

\begin{tabular}{|l|l|}
\hline \multirow{2}{*}{ Hemoterapia } & \multicolumn{1}{c|}{\begin{tabular}{c}
\multicolumn{1}{c|}{ Instituto Provincial de Hemoterapia; } \\
INCUCA
\end{tabular}} \\
\hline \multirow{5}{*}{ Perinatal } & $\begin{array}{l}\text { Dirección Nacional de SIDA y ETS; } \\
\text { Programa Provincial de HIV, enfermedades } \\
\text { transmisibles y hepatitis viral; }\end{array}$ \\
& $\begin{array}{l}\text { Programa Nacional de Salud Sexual y } \\
\text { Procreación Responsable; } \\
\text { Programa Provincial de Salud Reproductiva } \\
\text { y Procreación Responsable; } \\
\text { HIV/Infecciones de Transmisión Sexual } \\
\text { Nación; } \\
\text { Dirección de Materno Infancia de Nación; } \\
\text { Plan Nacional de Reducción de Mortalidad } \\
\text { Materno Infantil; } \\
\text { Redes Perinatales de Provincia; } \\
\text { Programa Nacional de Cardiopatías } \\
\text { Congénitas (PNCC) }\end{array}$ \\
\hline \multirow{5}{*}{ Nefrología } & $\begin{array}{l}\text { Programa de Prevención, Diagnóstico } \\
\text { y Tratamiento del Paciente Diabético } \\
\text { (PRODIABA); } \\
\text { Programas locales de hábitos saludables. }\end{array}$ \\
\hline
\end{tabular}

Fuente: elaboración del GTDGPS en base al relevamiento 2015

Los flujos de información que circulan en el interior de las redes (Blanco, 2009), abonan - en general- a la gestión de información financiera, clínica y epidemiológica (de la especialidad en la que trabaja cada una). Se utilizan para analizar casos particulares, resolver situaciones coyunturales, y, en algunas ocasiones, para proyectar intervenciones. El responsable de la red perinatal de la RS VI, manifestó que:

[...] en la época invernal, casi todos los lunes viene un rato acá [médico cirujano del equipo] para analizar juntos datos, hacemos presentaciones conjuntas de la información que generamos desde los dos lugares para analizar con los servicios al final de la época invernal, con todo lo que implica IRAB [...]. (Entrevista $n^{0} 14$ Red Perinatal)

En detrimento de una mejor planificación, pocas redes logran generar su propia información; no todas cuentan con el tiempo y los recursos necesarios para su producción. Un referente de la red de emergencias (Sies), nos proporciona un ejemplo del tipo de información que generan las redes de servicios estudiadas:

Clínica y epidemiológica sí, porque te puedo decir:en el año hemos tenido una incidencia de, no sé, tantos traumatismos de cráneos, con tales patologías, que fueron resueltos en tales lugares, eso sí [...] información epidemiológica también [...] de todo lo que es epidemiología en trauma, por ejemplo. (Entrevista $\mathrm{n}^{0} 13$ Red SIES)

El padrón que es una herramienta organizacional necesaria, tanto para efectores como para organismos de salud, pocas veces es utilizada para organizar las intervenciones en relación a las características de la población objetivo. Las redes que cuentan con un sistema de nominalización, al no existir un único padrón nacional, provincial o municipal, utilizan varios de manera simultánea, en general diseñados por problemática. Se identificaron nueve padrones $^{14}$ diferentes, siendo el RESAPRO y el SUMAR los más usados. Esto evidencia un avance sobre la nominalización de la población, pero a la vez muestra una distancia de la propuesta del Plan Federal de consolidar un Padrón Único de Salud (PAS) que incluya las bases de seguridad social, prepagas y CAPS en un solo registro (Plan Federal de Salud 2004-2007).

En un contexto de escasas políticas de macrogestión, la organización en red presenta cierto progreso para el sector en términos de acceso a la atención, aunque aún persisten diversas dificultades organizacionales asociadas a la falta de madurez de cada red. Sobresalen en los relatos cuestiones como: la mala comunicación y/o comunicación no informatizada (muchas veces por falta Internet); poca sistematización y registro de las actividades; carencia de designaciones formales del staff (que otorguen entidad a la red); ausencia de presupuesto exclusivo para obtener mayor grado de autonomía; fortalecimiento de la referencia y contrarreferencia que permita incrementar y mejorar el registro

14 RESAPRO: Registro Sanitario Provincial/ SUMAR: registro programa SUMAR/ SIISA: Sistema Integrado de Información Sanitaria Argentina/ SIVILA: Sistema Nacional de Vigilancia de la Salud/ SIPerinatal: Sistema de Información Perinatal/ Prodiaba: registro del Programa de Prevención, Diagnóstico y Tratamiento del Paciente Diabético/ Remediar: registro programa Remediar/ SITAM: Sistema de Información para el Tamizaje. 
de la información sobre el paciente (historias clínicas completas y claras); y optimización de las camas para lograr disponibilidad en momentos críticos, como el periodo invernal. Las palabras del coordinador de una red de salud sexual y procreación responsable (SSyPR) refieren, específicamente, a las limitaciones presupuestarias que experimentan las redes en general:

También de hecho, yo tengo un gran compromiso. Me emociona, te lo estoy contando y digo tantas cosas ¿No? Pero bueno, a veces me enoja esto de que no haya un presupuesto, no haya un café o que tenga que pagarde mi bolsillo. Aveces digo, que harían sin mísi no tuviera auto. (Entrevista $\mathrm{n}^{0} 22$ Red SSyPR)

Otros problemas que afectan a la dimensión organizacional replican los propios del sector: un primer nivel de atención poco fortalecido, que además cuesta priorizar en relación al hospital, lo que muchas veces dificulta llegar a la población de referencia y comprender sus necesidades; la falta de camas sobre todo en áreas críticas (pediátricas, unidades intensivas de adulto, etc.); la escasez y/o mala distribución geográfica de profesionales de distintas especialidades (médicas pero también técnicas); insuficientes sistemas de información e historias clínicas informatizadas que agilicen la atención, etc. En particular, las dificultades del primer nivel para brindar una atención adecuada a las necesidades de la población han sido expresadas por la mayoría de los testimonios recogidos. Los argumentos del referente de una red de enfermedades crónicas no transmisibles, por ejemplo, alertan sobre esta problemática:

[...] habría que fortalecer la formación en atención primaria para que haya una oferta más cercana al paciente y a la atención digamos. Lo que eso lleva después a modificar la coordinación de que llegue la medicación al centro de salud. Por eso también es una decisión muy local (municipal). (Entrevista $\mathrm{n}^{0}{ }_{10}$ Red ECNT)

\section{La articulación de los servicios en el territorio}

Las redes estudiadas muestran diferentes escalas territoriales: mientras algunas son nacionales o provinciales, otras se despliegan al interior de las regiones sanitarias. Esto da cuenta de los desafíos diferenciales que tienen las redes en la gestión de sus áreas programáticas ${ }^{15}$ (Ramírez, 2004), tanto en términos de las responsabilidades que cada participante tiene, como de las interacciones que suponen las relaciones entre actores de diversos niveles jurisdiccionales. Además, establecer dicha área les ha permitido a las redes planificar intervenciones en función de las necesidades y demandas de su territorio, aunque, el mayor problema consiste en que no necesariamente tienen correlato con los modos en que la población se moviliza para atenderse. Al respecto un entrevistado ha sostenido que:

[...] porun lado las áreas programáticas tienen el gran problema de serun cálculo teórico sobre la realidad, después la realidad te confronta y la gente no se mueve como uno quisiera [...] en realidad uno lo tiene que concebir de una manera mucho más dinámica ¿no? Alguna base de cálculo tenés que tener [...] tiene sus lógicas y una mismaárea programática quizás te sirve para una cosay [para otra no]. (Entrevista $n^{\circ} 8$ Red Perinatal)

Por otro lado, aquellas redes que abordan problemáticas de salud que involucran articulaciones con el primer nivel de atención presumen una dinámica territorial más compleja. Las interacciones que se tejen entre la red y el territorio de proximidad (que alberga al Centro de Salud) suponen decodificar la trama singular del espacio local (lo que no significa que dichas interacciones sean siempre virtuosas). La complejidad que caracteriza a los vínculos entre instituciones de distintos niveles de atención dentro de las redes, ha sido señalada por uno de los referentes de la red provincial de emergencias (SIES):

15 Todas las redes tienen definidas formalmente sus áreas programáticas. La única excepción es la red de Nefrología, posiblemente debido a la baja prevalencia de pacientes en Diálisis Crónica en la población. 
[...] Bueno, si uno habla de las características sociales dentro de lo que es la región, tenemos un polimorfismo terrible porque tenemos áreas [...] muy densamente pobladas como es Lanús, o sea el primer cordón -porque llegamos hasta el tercer cordón del conurbano nosotros. Donde tenés un tipo de realidad, un tipo de paciente; hasta zonas donde, por ejemplo Ezeiza, donde tenés una densidad de población muy muy baja, ya casi un área rural digamos. En ese sentido, tenemos una población muy diferente. (Entrevista ${ }^{0} 17$ Red SIES)

Con el propósito de integrar a los establecimientos como corresponsables en los procesos de atención de una población determinada, las redes despliegan ciertos mecanismos y dispositivos para articular recursos, prestaciones y servicios dispersos en el territorio, a partir de acuerdos -con distintos grados de formalidaden los que las partes puedan exigirse mutuamente.

El padrón, presentado más arriba como una herramienta organizacional relevante, es también un dispositivo clave para la articulación de los servicios, aunque en la mayoría de las redes se encuentra subutilizado, y acaba perdiéndose la posibilidad de cuantificar y georreferenciar las demandas de la población según su perfil.

Otro de esos dispositivos alude a un espacio interinstitucional - en términos de Hintze (2009) -, con un responsable de coordinar los recursos (físicos y humanos) y los insumos que circulan, o las actividades que desarrollan. En nuestro estudio, ese ámbito no siempre fue identificado, y en determinadas ocasiones tampoco pudo precisarse quiénes lo integraban o qué recursos estaban disponibles. Esto configura situaciones de baja capacidad institucional $^{16}$ (Tobelem, 1993) en algunas redes, que se expresa, por ejemplo, en la falta de un espacio físico para la coordinación y de personal e insumos formalmente designados. También, esto ha sido interpretado como parte de las dificultades más generales de la macro política sanitaria en la implementación de un marco regulatorio específico para la gestión de estas redes. Por ejemplo, en la entrevista realizada al coordinador de una red perinatal, se mencionan distintas instancias en las cuales la falta de políticas nacionales o subnacionales para la gestión en red de los servicios se pone al descubierto:

[...] no tenemos elementos concretos de gestión, no podemos definirmovilización, movilidad depersonal, por ejemplo, o de otro tipo de recursos [...] eso está asociado [al] hecho de que no tenemos leyes [...] que configuren un sistema de atención de salud en función de las redes. (Entrevista $n^{\circ} 8$ Red Perinatal)

Cuando el espacio interinstitucional se localiza formalmente en algún establecimiento de la red o en la propia oficina de la región sanitaria, las reuniones entre sus integrantes ocurren de manera sistemática y frecuente. A la vez, su composición revela dos posibles alternativas: una en la que encontramos pares profesionales de distintas especialidades que, con ciertos niveles de autonomía, comparten la gestión de la red (por ejemplo, neonatólogos, pediatras, cardiólogos infantiles) con el apoyo de un equipo de técnicos y/o administrativos; otra en la que sólo hay un profesional (de conocimiento experto) a cargo con un reducido staff técnico y/o administrativo que experimenta una sobrecarga importante de responsabilidades.

Contrariamente, en los casos en los que el espacio no se ha formalizado, las oportunidades de encuentro entre los miembros son más improvisadas y esporádicas. Esto redunda en dificultades para implementar mecanismos acordados que optimicen el manejo de la información, la planificación de las actividades y la utilización de los recursos disponibles, comprometiendo la organización y el desempeño de la misma red.

Otra de las formas en que se expresa esa baja capacidad institucional, se refiere a la ausencia de una designación formal de profesionales como responsables de la coordinación. En ocasiones, son los propios referentes de los programas - sobre los que se apoya la red- los que terminan desarrollando

16 Tobelem (1993) ha planteado que la capacidad institucional presenta cinco grandes dimensiones: (1) la institucional propiamente dicha, relacionada con normas y reglas de juego que condicionan la ejecución de tareas; (2) la de coordinación interorganizacional e interjurisdiccional; (3) la de organización interna y asignación de tareas; (4) la de capacidad financiera y física de la organización; y (5) la vinculada a los sistemas de recursos humanos, políticas de personal e incentivos. 
esta tarea. Sin embargo, a pesar de los matices encontrados respecto de la designación, todos los entrevistados indicaron percibir una remuneración por sus actividades ${ }^{17}$ (aunque no vinculada a un cargo específico por el trabajo realizado en la red).

La sistematización y clasificación de las actividades desarrolladas por los coordinadores (Cuadro 3) arroja que las vinculadas a la articulación ${ }^{18}$ y a la gestión aparecen como las más recurrentes, mientras que la vigilancia, el control y la capacitación se presentan como de menor relevancia; paralelamente, el manejo de la información ocupa el lugar más residual o marginal. Llama la atención la centralidad que adquieren ciertas tareas que se transforman en mecanismos operativos para el funcionamiento de la red - llevados a cabo por los propios coordinadores ${ }^{19}$-, asociados a la situación de precariedad que afrontan muchos de los servicios. Este tipo de trabajo refiere generalmente a cuestiones cotidianas de resolución inmediata para sortear algún obstáculo puntual, más que a la gestión. Se han mencionado iniciativas informales (Crojethovic, 2012) ${ }^{20}$ para resolver problemáticas que deben ser atendidas con urgencia, como la gestión de camas o la derivación de pacientes. Así, el desempeño de cada red depende de un alto compromiso por parte de los actores involucrados.

\section{Cuadro 3 - Actividades de los referentes}

\begin{tabular}{|c|c|c|c|c|c|}
\hline De gestión & $\begin{array}{l}\text { Vigilancia y } \\
\text { control }\end{array}$ & Articulación & Capacitación & Información & Informales \\
\hline $\begin{array}{l}\text { Gestionar las } \\
\text { actividades de } \\
\text { promoción y } \\
\text { prevención }\end{array}$ & $\begin{array}{c}\text { Vigilancia para el } \\
\text { cumplimiento de } \\
\text { normas }\end{array}$ & $\begin{array}{l}\text { Vincular a los } \\
\text { jefes de servicios }\end{array}$ & $\begin{array}{l}\text { Capacitación } \\
\text { de los recursos } \\
\text { profesionales }\end{array}$ & $\begin{array}{l}\text { Manejo de la } \\
\text { base de datos, } \\
\text { generación } \\
\text { de usuarios y } \\
\text { habilitaciones. }\end{array}$ & $\begin{array}{l}\text { "Patear los } \\
\text { municipios" }\end{array}$ \\
\hline $\begin{array}{c}\text { Gestión y } \\
\text { distribución } \\
\text { de insumos } \\
\text { (sobre todos } \\
\text { medicamentos) }\end{array}$ & Control insumos & $\begin{array}{l}\text { Articular con } \\
\text { distintos } \\
\text { programas }\end{array}$ & Dictado de cursos & $\begin{array}{c}\text { Coordinar } \\
\text { campañas en } \\
\text { hospitales o en el } \\
\text { territorio }\end{array}$ & $\begin{array}{c}\text { Recepción y } \\
\text { control de los } \\
\text { casos (pacientes) }\end{array}$ \\
\hline $\begin{array}{c}\text { Logística general } \\
\text { de la red }\end{array}$ & $\begin{array}{l}\text { Gestionar los } \\
\text { operativos de } \\
\text { control }\end{array}$ & $\begin{array}{c}\text { Articular con } \\
\text { otros actores } \\
\text { (ONGS, empresas, } \\
\text { etc.) }\end{array}$ & $\begin{array}{l}\text { Capacitación } \\
\text { en servicios, } \\
\text { escuelas o a } \\
\text { docentes. }\end{array}$ & & $\begin{array}{l}\text { Resolución de } \\
\text { casos puntuales }\end{array}$ \\
\hline $\begin{array}{l}\text { Gestionar la } \\
\text { comunicación } \\
\text { sobre la } \\
\text { problemática } \\
\text { abordada }\end{array}$ & $\begin{array}{c}\text { Monitoreo y } \\
\text { evaluación } \\
\text { de cantidad } \\
\text { y calidad de } \\
\text { establecimientos }\end{array}$ & $\begin{array}{c}\text { Articular } \\
\text { prácticas al } \\
\text { interior de la red }\end{array}$ & & & Recibir denuncias \\
\hline $\begin{array}{l}\text { Gestionar } \\
\text { estudios } \\
\text { requeridos en } \\
\text { casos especficos }\end{array}$ & & $\begin{array}{l}\text { Articular con } \\
\text { los referentes } \\
\text { municipales y/o } \\
\text { de hospitales }\end{array}$ & & & Gestión de camas \\
\hline
\end{tabular}

continúa...

17 Con excepción del coordinador de la red de SSyR.

18 Las referencias explícitas a "la articulación de la red" no siempre tienen el mismo sentido en la voz de nuestros entrevistados. Puede denotar la articulación entre pares, entre distintos servicios o entre diferentes niveles de complejidad. También la coordinación entre otras instituciones que no forman parte del sector, o bien entre actores y organizaciones que pertenecen a otros niveles jurisdiccionales.

19 Otros autores como Mintzberg (2005), también han encontrado organizaciones complejas en donde los profesionales o expertos destinan parte importante de su tiempo al desarrollo de actividades administrativas.

20 Lo informal representa un proceso complejo que se entreteje con lo formal de diversas formas constituyéndose como parte del desarrollo organizacional. Dentro de lo informal, las iniciativas de mediano y largo plazo posibilitan el cambio organizacional, mientras que las de corto plazo desvían a la organización de sus objetivos reproduciendo muchas veces la precariedad del sistema (Crojethovic, 2012). 


\section{Cuadro 3 - Continuación}

\begin{tabular}{|c|c|c|c|c|c|}
\hline De gestión & $\begin{array}{l}\text { Vigilancia y } \\
\text { control }\end{array}$ & Articulación & Capacitación & Información & Informales \\
\hline $\begin{array}{l}\text { Clasificación de } \\
\text { los hospitales } \\
\text { integrantes } \\
\text { según su } \\
\text { complejidad de } \\
\text { atención }\end{array}$ & & $\begin{array}{c}\text { Articular entre } \\
\text { niveles }\end{array}$ & & & $\begin{array}{l}\text { Tarea diaria } \\
\text { de gestión } \\
\text { telefónica/ } \\
\text { derivación de } \\
\text { pacientes }\end{array}$ \\
\hline $\begin{array}{l}\text { Gestión de } \\
\text { cargos de } \\
\text { personal }\end{array}$ & & Armar subredes & & & $\begin{array}{c}\text { Resolución } \\
\text { de problemas } \\
\text { puntuales } \\
\text { intrahospitalarios }\end{array}$ \\
\hline \multirow[t]{2}{*}{$\begin{array}{c}\text { Gestionar las } \\
\text { interacciones } \\
\text { con el nivel más } \\
\text { local }\end{array}$} & & $\begin{array}{c}\text { Articulación con } \\
\text { Nación }\end{array}$ & & & \\
\hline & & $\begin{array}{c}\text { Articular con los } \\
\text { otros sistemas de } \\
\text { salud (privados: } \\
\text { abiertos y } \\
\text { cerrados) }\end{array}$ & & & \\
\hline
\end{tabular}

Fuente: elaboración del GTDGPS en base al relevamiento 2015

En suma, el trabajo de los coordinadores en la gestión deja entrever no sólo la diversidad de asuntos y actividades que implica su funcionamiento, sino también los altos grados de informalidad y -nuevamente- la baja capacidad institucional que caracteriza a estas experiencias.

\section{Consideraciones finales}

El modo de organización en red de los servicios de salud apareció en la Argentina durante la última década, como estrategia para mejorar los resultados en el acceso, la cobertura y los beneficios en salud de la atención pública. Obtener datos para medir el impacto de dicha política sanitaria es complejo, aunque pudimos inferir cierto avance en las decisiones políticas y en las acciones orientadas a la coordinación de niveles en el proceso de atención, que procuraron incrementar el acceso de la población a las prestaciones.

Las redes promovieron la ampliación de la escala de las intervenciones, facilitando la circulación de ciertos insumos e información entre establecimientos, y vinculando territorios sobre todo de escala regional, con distintas capacidades y equipamientos para responder a las necesidades de salud. A pesar de esto, el desarrollo de la nueva estrategia organizacional no consiguió vincular a los tres subsectores y no logró desarticular la lógica hospitalocéntrica, debido a que reprodujeron la baja capacidad institucional del subsector. Así, la carencia de instancias, dinámicas y recursos formalmente destinados a un adecuado desempeño de las redes ha impuesto importantes limitaciones para su desarrollo y autonomía, lo que ha implicado un alto compromiso y un gran esfuerzo por parte de los equipos involucrados para poder gestionarlas.

\section{Referencias}

ACUÑA, C.; CHUDNOVSKY, M. El Sistema de Salud en Argentina. Buenos Aires: Universidad de San Andrés, 2002. Documento 60.

ANDERSEN, R. A. Revisiting the behavioral model and access to medical care: does it matter? Journal of Health and Social Behavior, Washington, DC, v. 36, n. 1, p. 1-10, 1995.

ANDIARENA, S. La organización en red para la atención de las cardiopatías congénitas: una 
experiencia de coordinación interinstitucional. La Rivada, Misiones, v. 6, n. 11, p. 61-8o, 2018,

BLANCO, J. Redes y territorios: articulaciones y tensiones. In: ENCUENTRO DE GEÓGRAFOS DE AMÉRICA LATINA, 12., 2009, Montevidéu. Anais... [S.l.]: Egal, 2009.

CETRÁNGOLO, O. Financiamiento fragmentado, cobertura desigual y falta de equidad en el sistema de salud argentino. Revista de Economía Política de Buenos Aires, Buenos Aires, v.8, n. 13, p. 145-183, 2014.

CHIARA, M. ¿Restricciones o incentivos? Revisando las tensiones del contexto desde una caracterización del régimen de implementación. In: CHIARA, M. (Comp.). Salud, política y territorio en el Gran Buenos Aires. Los Polvorines: Ed. UNGS, 2012. p. 67-109.

CHIARA, M.; ARIOVICH, A. Luces y sombras sobre el territorio. Reflexiones en torno a los planteamientos de la OPS /OMS en América Latina. Cadernos Metropole, São Paulo, v. 15, n. 29, p. 99-122, 2013.

CHIARA, M.; Di VIRGILIO, M.; ARIOVICH, A. La coparticipación provincial por salud en el Gran Buenos Aires: reflexiones en torno a la política local (2000-2005). Salud Colectiva, Buenos Aires, v. 6, n. 1, p. 47-64, 2010.

CROJETHOVIC, $\mathrm{m}$. El aspecto informal de las organizaciones públicas: instrumento de dominación y estrategia para el cambio. Cuadernos del CENDES, Venezuela, v. 29, n. 79, 2012.

CROJETHOVIC, M. Universalismo en salud. La reconstrucción de un debate sobre los derechos. Revista Via Iuris, Bogotá, DC, n. 21, p. 13-23, 2016. CROJETHOVIC, M.; Ariovich, A. Las redes: un modelo organizativo para contrarrestar la fragmentación institucional del sistema de salud en la Argentina. Revista Gestión de las Personas y Tecnología, Santiago, n. 24, p. 30-50, 2015.

CURSIO, J. Descripción del sistema de seguridad social: componentes al cabo de la década del '9o y de la primera década del siglo XXI. In: DANANI, C.; HINTZE, S. (Comp.). Protecciones y desprotecciones: la seguridad social en la Argentina 1990-2010. Los Polvorines: Ed. UNGS, 2011.

DABAS, E.; PERRONE, N. Redes en Salud. Buenos Aires: [s.n.], 1999. Disponível em: <https://bit. ly/zeCFhwW>. Acesso em: 1 jun. 2020.

DONABEDIAN, A. An introduction to quality assurance in health care. Nova York: Oxford University Press, 2003.

ETZIONI, A. Organizaciones modernas. Buenos Aires: UTHEA, 1975.

FLEURY, S. El desafío de la gestión de las redes de políticas. Revista Instituciones y Desarrollo, Barcelona, n. 12-13, p. 221-247, 2002.

GUTIÉRREZ, A. La movilidad de la metrópolis desigual: el viaje a la salud pública y gratuita en la periferia de Buenos Aires. In: ENCUENTRO DE GEÓGRAFOS DE AMÉRICA LATINA, 12., 2009, Montevidéu. Anais... [S.l.]: Egal, 2009.

HARZHEIM, E. Atenção primária à saúde e as redes integradas de atenção a saúde. In: HARZHEIM E. (Ed.). Inovando o papel da atenção primária nas redes de atenção à saúde: resultados do laboratório de inovação em quatro capitais brasileiras. Brasília, DF: Organização PanAmericana da Saúde, 2011. p. 45-54.

HINTZE, J. Modelos organizativos para la gestión social y sus lógicas. In: CHIARA, M.; DI VIRGILIO, M. (Org.). Gestión de política social. Conceptos y herramientas. Buenos Aires: Prometeo, 2009. p. 87-118.

LEVESQUE, J. F.; HARRIS, M. F.; RUSSELL, G. Patient-centred access to health care: conceptualising access at the interface of health systems and populations. International Journal for Equity in Health, Londres, v. 12, n. 1, p. 12-18, 2013.

MENDES, E. V. Las redes de atención de salud. Brasília, DF: OPS, 2013.

MINTZBERG, H. La estructuración de las organizaciones. Madri: Ariel, 2005.

OPS - ORGANIZACIÓN PANAMERICANA DE LA SALUD. Salud en las Américas 2007. Volume I Regional. Washington, DC, 2007. 
OPS - ORGANIZACIÓN PANAMERICANA DE LA SALUD. Sistemas integrados de servicios de salud. Conceptos, opciones de política y hoja de ruta para su Implementación en las Américas. Washington, DC, 2008.

OPS - ORGANIZACIÓN PANAMERICANA DE LA SALUD. Estrategia para el acceso universal a la salud y la cobertura universal de salud. Washington, DC, 2014.

RAMÍREZ, L. Determinación de áreas de influencia hospitalaria mediante análisis espacial en SIG vectorial: un aporte metodológico. In: SEMINARIO ARGENTINO DE GEOGRAFÍA CUANTITATIVA, 1., 2004, Buenos Aires. Anais... [S.l. : s.n.], 2004. Disponível em: <https://bit. ly/2zGewZP>.

Acesso em: 1 jun. 2020.

SALVIA, A. et al. La salud de la población y el acceso al sistema que la atiende In: SALVIA, A. et al.
Estado de situación del desarrollo humano y social: barreras estructurales y dualidades de la sociedad argentina en primer año del bicentenario. Buenos Aires: Educa, 2011. p. 135-175.

SHORTELL, S. et al. Creating organized delivery systems: the barriers and facilitators. Hospital \& Health Services Administration, [S.l.], n. 38, p. 447-466, 1993.

TOBELEM, A. Sistema de análisis y desarrollo de la capacidad institucional (SADCI). Manual de Operaciones. Publicaciones Ocasionales de la División de Administración del Sector Público del Departamento Técnico de la Región América Latina y Caribe del Banco Mundial, 1993.

VIANNA, M. L. T. W. A americanização (perversa) da seguridade social no Brasil: estratégias de bem-estar e políticas públicas. Rio de Janeiro: Revan, 200o.

\section{Contribución de los autores}

Las autoras trabajaron con igual nivel de responsabilidad en las tareas de relevamiento, procesamiento, análisis y escritura del documento.

Recibido: 29/11/2019

Re-presentado: 31/03/2020

Aprobado: 15/04/2020 